\title{
Editorial
}

\section{Antenna Design for Microwave and Millimeter Wave Applications: Latest Advances and Prospects}

\author{
Hosung Choo (1)
}

Citation: Choo, H. Antenna Design for Microwave and Millimeter Wave Applications: Latest Advances and Prospects. Appl. Sci. 2021, 11, 5556. https://doi.org/10.3390/app11125556

Received: 6 June 2021

Accepted: 11 June 2021

Published: 16 June 2021

Publisher's Note: MDPI stays neutral with regard to jurisdictional claims in published maps and institutional affiliations.

Copyright: (C) 2021 by the author. Licensee MDPI, Basel, Switzerland. This article is an open access article distributed under the terms and conditions of the Creative Commons Attribution (CC BY) license (https:/ / creativecommons.org/licenses/by/ $4.0 /)$.
Department of Electronic and Electrical Engineering, Hongik University, Seoul 04066, Korea; hschoo@hongik.ac.kr

\section{Introduction}

Until recently, substantial effort has been devoted to new approaches and attempts to the design of antennas for microwave and millimeter-wave applications. For example, advanced technologies such as antenna miniaturization, array optimization, and bandwidth enhancement have been extensively studied over a decade, and are being applied to commercial applications such as 4G/5G mobile communications, autonomous driving, or military applications including radar, direction finding, and anti-jamming. However, as these technologies were recently employed in small mobile devices, the size and geometry of the antennas are more limited in order to be mounted in a more compact space with better radiation performances. Accordingly, advanced antenna designs using novel approaches to this issue are required in various aspects. This Special Issue aims to collect relevant papers describing the latest advances and prospects in antenna design for microwave and millimeter-wave applications. The fields of interest for this Special Issue include, but are not limited to, design methods of antennas such as miniaturization, optimization, and array. In total, 22 papers were submitted and 14 of them were published in the Special Issue. The following section provides brief summaries of each published paper.

\section{Design of Heterogenous Two-Element Array Antenna on an Electrically Thick Substrate for High Isolation and Low Pattern Correlation Using Modal Difference in Radiation Patterns}

Lim et al. [1] proposed a novel design of a two-element array antenna on an electrical thick substrate with an extremely narrow array distance. The proposed array consists of a rectangular ring patch printed on a thick substrate and a monopole wire in the center of the substrate. Each element has a modal difference in the radiation pattern, causing high isolation and low correlation between the array elements. From the measurement results, the monopole and patch elements exhibited reflection coefficients of $-10 \mathrm{~dB}$ and $-10.7 \mathrm{~dB}$ with peak gains of $3.8 \mathrm{dBi}$ and $6.1 \mathrm{dBi}$, respectively, at $1.6 \mathrm{GHz}$. The mutual coupling between the two elements was $-20.7 \mathrm{~dB}$. For modal analysis of the antenna pattern, spherical mode decomposition was performed on the radiation patterns of the two elements, and low envelope correlation coefficient levels below 16\% were maintained. The anti-jamming performance using a power inversion algorithm was also investigated; a null depth of $-47.7 \mathrm{~dB}$ and a null width of $33.2^{\circ}$ were obtained when the interference signal arrived at the elevation angle of $45^{\circ}$.

\section{A Novel High Gain Monopole Antenna Array for 60 GHz Millimeter- Wave Communications}

Mneesy et al. [2] presented the design and implementation of a $60 \mathrm{GHz}$ single element monopole antenna as well as a two-element array made of two $60 \mathrm{GHz}$ monopole antennas. The proposed antenna array was used for $5 \mathrm{G}$ applications with radiation characteristics that conformed to the requirements of wireless communication systems. The proposed single element was designed and optimized to work at $60 \mathrm{GHz}$ with a bandwidth of 
6.6 GHz $(57.2-63.8 \mathrm{GHz})$ and a maximum gain of $11.6 \mathrm{dBi}$. The design was optimized by double T-shaped structures that were added in the rectangular slots, as well as two external stubs in order to achieve a highly directed radiation pattern. Moreover, ring and circular slots were made in the partial ground plane at an optimized distance as a defected ground structure (DGS) to improve the impedance bandwidth in the desired band. The two-element array was fed by a feed network, thus improving both the impedance bandwidth and gain. The single element and array were fabricated, and the measured and simulated results mimicked each other in both return loss and antenna gain.

4. Method for Estimating Optimal Position of S-Band Relay Station through Path Loss Analysis in an Outdoor Environment

Jang et al. [3] investigated a novel estimation method to optimize the position of a relay station of the S-band telemetry system in outdoor environments. To determine an optimal relay station position, complex terrain surface and buildings near the Naro Space Center were included in the electromagnetic (EM) simulation. The optimal relay station position was then determined using the estimation method by comparing the path losses from the rocket to the main station and some candidate positions of the relay station. To better understand the path loss, the ray analysis was also conducted, and the result demonstrated that once the rocket altitude to be covered by the relay station is determined, it is possible to find the optimum position of the relay station.

5. Numerical Study on the Feasibility of a $24 \mathrm{GHz}$ ISM-Band Doppler Radar Antenna for Near-Field Sensing of Human Respiration in Electromagnetic Aspects

In this paper [4], the feasibility study of a $24 \mathrm{GHz}$ industrial, scientific, and medical (ISM) band Doppler radar antenna in electromagnetic aspects was numerically performed for near-field sensing of human respiration. The Doppler radar antenna consists of a transmitting (Tx) antenna and a receiving ( $R x)$ antenna close to the human body for a wearable device. The designed slot-type Doppler radar antenna was embedded between an RO4350B superstrate and an FR-4 substrate. To obtain the higher radiation pattern of the antenna towards the human body, a ground plane reflector was placed underneath the substrate. The measured $-10 \mathrm{~dB}$ reflection coefficient (S11) bandwidth was 23.74 to $25.56 \mathrm{GHz}$ and the mutual coupling (S21) between Tx and Rx antennas was lower than $-30 \mathrm{~dB}$ at target frequencies. The Doppler effect due to human respiration was investigated through the I/Q and arctangent demodulation of the returned signal. According to the results, the phase variation of the returned signal was proportional to the displacement of the body's surface, which was about $0.8 \mathrm{rad}$ in accordance with $1 \mathrm{~mm}$ displacement. The numerical experiments indicated that the proposed Doppler radar antenna can be used for near-field sensing of human respiration in electromagnetic aspects.

6. Design of an Electrically Small, Planar Quasi-Isotropic Antenna for Enhancement of Wireless Link Reliability under NLOS Channels

The performance of wireless networks can be greatly influenced by the radiation pattern and polarization of the antennas at the nodes, especially when they are under non-line-of-sight (NLOS) channel environments. In this paper [5], a planar quasi-isotropic antenna based on the combination of a meandered electric dipole and an electrically small loop was designed at a frequency of $2.45 \mathrm{GHz}$. Its electrical size $(\mathrm{ka})$ was 0.47 and showed a gain deviation of $3.01 \mathrm{~dB}$ with a radiation efficiency of $82.6 \%$ per the simulation. The performance of a wireless link under the line-of-sight and NLOS channels in an indoor environment was measured using the proposed quasi-isotropic antenna as a receiving antenna after validating its radiation and impedance properties experimentally (the measured gain deviation: $5.2 \mathrm{~dB}$, the measured radiation efficiency: $79.2 \%$ ). This study demonstrated that better properties were achieved using the quasi-isotropic antenna. The quasi-isotropic antenna showed an improved packet delivery ratio (PDR) and received signal strength indicator (RSSI) compared to the results using omni-directional antennas as a transmitting and receiving pair in the NLOS channels. 


\section{Software-Defined Radio Beamforming System for 5G/Radar Applications}

Based on the flexibility of software-defined radio (SDR) techniques applied to an array of antennas, this paper [6] presented a beamforming architecture designed to operate in millimeter-wave bands $(28 \mathrm{GHz})$, with possible applications in radar and 5G systems. The system structure, including its main constituents such as the radio frequency (RF) front-end modules, the radiating elements as well as the baseband processing on the host computer, were widely described. Beamforming was achieved by digitally controlling the signals that fed the antennas. The experimental measurements performed in an anechoic chamber validated the proposed approach.

\section{Design of a Monopulse System Using a Single Patch Radiator with a Simple Multi-Mode Substrate Integrated Waveguide Feeding Network}

Youn et al. [7] proposed the design of a single radiator monopulse antenna with a substrate integrated waveguide (SIW) multi-mode feeding system to reduce the aperture size and simplify the feeding structure. The proposed antenna can effectively reduce the overall system size by using a single patch radiator, compared to conventional systems that require multiple radiators. In addition, to simplify the structure of the pattern comparator, an SIW multi-mode feeding system with $\mathrm{TE}_{10}$ and $\mathrm{TE}_{20}$ modes was used. To verify the feasibility, the patch antenna with the SIW multi-mode feed was fabricated, and its antenna characteristics of reflection coefficients, mutual coupling, and radiation patterns were measured in a full anechoic chamber. The accuracy of the direction of arrival (DOA) estimation was further improved by applying the derived calibration factor to the resulting sum and difference ratio. The results demonstrated that the proposed antenna is suitable for compact and lightweight monopulse systems with the single radiator and the simple multi-mode feeding network.

\section{Quasi-Yagi Slotted Array Antenna with Fan-Beam Characteristics for 28 GHz 5G Mobile Terminals}

Kim et al. [8] proposed a quasi-Yagi slotted array antenna with fan-beam characteristics for $28 \mathrm{GHz} 5 \mathrm{G}$ mobile terminals. The antenna was composed of a $1 \times 8$ slot antenna array with directors to enhance the half-power beamwidth (HPBW). The proposed antenna had a fan-beam radiation pattern with a simulated HPBW of $256.72^{\circ}$ and a peak gain of $11.16 \mathrm{dBi}$. In addition, the proposed antenna covered $\pm 48^{\circ}$ using a beam steering mechanism. Mutual coupling reduction was achieved by inserting slits between the adjacent slot radiators on the ground plane. The simulated $-10 \mathrm{~dB}$ reflection coefficient bandwidth of the proposed antenna was $1.79 \mathrm{GHz}(27.03-28.82 \mathrm{GHz})$, and the mutual coupling between each of the slot radiators was lower than $-25.02 \mathrm{~dB}$ over the $28 \mathrm{GHz}$ target band $(27.5-28.35 \mathrm{GHz})$. To investigate the effect of a human body in a practical environment, the power density was considered to estimate the electromagnetic exposure with a simplified skin model. The measured results were in good agreement with the simulated ones and demonstrated that the proposed antenna could be used for $5 \mathrm{G}$ mobile terminals.

\section{Low-Profile Spidron Fractal Dipole Antenna with a Ferrite-Loaded Artificial Magnetic Conductor for Manpack Applications}

Kwon et al. [9] presented a Spidron fractal dipole antenna with a ferrite-loaded artificial magnetic conductor (AMC). By applying ferrite composed of nickel-zinc with a high permeability value, a compact AMC operating in the broadband frequency range within the high-frequency/very-high-frequency/ultra-high-frequency (HF/VHF/UHF) bands was designed. A Spidron fractal-shaped dipole antenna with a quasi-self-complementary structure was designed and combined with a miniaturized ferrite-loaded AMC. This allowed the designed AMC-integrated dipole antenna to operate in a wide frequency band, covering the HF/VHF/UHF bands, with low-profile characteristics. A prototype of the proposed Spidron fractal dipole antenna with the AMC was manufactured and measured and found to meet low voltage standing wave radios (VSWR) specifications of $<3.5$ within the 20 $500 \mathrm{MHz}$ bandwidth range. The simulated and measured results were in good agreement. 
The size of the Spidron fractal dipole antenna with the AMC was $0.03 \times 0.026 \times 0.001 \lambda^{3}$ relative to the wavelength of the lowest operating frequency. The received power of the Spidron fractal dipole antenna with the AMC was also measured when it was applied to relatively small applications, such as a manpack in this case.

\section{Inverted F Type Antenna Design for the Cattle Activity and Estrus Detection Sensor Module}

Chung [10] designed and optimized an inverted F type antenna (IFA) for ZigBee communication of a sensor board. The designed antenna can replace the chip antenna on a radio frequency (RF) module that is not performing well enough for the ZigBee communication. The sensor board detects cattle behavior and identifies the breeding (estrus) period and transmits the data to the main station by the RF module and IFA antenna. The proposed and optimized transmitting/receiving (TRx) IFA antenna of the ZigBee communication module had a return loss of $-19 \mathrm{~dB}$ and a gain of $1.6 \mathrm{dBi}$ at $2.45 \mathrm{GHz}$. The size was about $2.5 \times 0.5 \mathrm{~cm}$ in width and vertical length, and the height was $0.55 \mathrm{~cm}$. The strengths of signals with the chip antenna and the IFA antenna have been measured and compared. There was about a $20 \mathrm{~dB}$ enhancement with the IFA antenna compared to the chip antenna. The antenna was designed and applied to the RF transmission and reception (TRx) module. This antenna and sensor module can be applied to livestock in general as well as cattle.

\section{Prediction of Wide Range Two-Dimensional Refractivity Using an IDW Interpolation Method from High-Altitude Refractivity Data of Multiple Meteorological Observatories}

Wang et al. [11] proposed a method for the prediction of wide range two-dimensional refractivity for synthetic aperture radar (SAR) applications, using an inverse distance weighted (IDW) interpolation of high-altitude radio refractivity data from multiple meteorological observatories. The radio refractivity was extracted from an atmospheric data set of twenty meteorological observatories around the Korean Peninsula along a given altitude. Then, from the sparse refractive data, the two-dimensional regional radio refractivity of the entire Korean Peninsula was derived using the IDW interpolation, in consideration of the curvature of the Earth. The refractivities of the four seasons in 2019 were derived at the locations of seven meteorological observatories within the Korean Peninsula, using the refractivity data from the other nineteen observatories. The atmospheric refractivities on 15 February 2019 were then evaluated across the entire Korean Peninsula, using the atmospheric data collected from the twenty meteorological observatories. The proposed IDW interpolation had the lowest average root-mean-square error (RMSE) of $\nabla \mathrm{M}$ (gradient of M), and more continuous results than other methods. To compare the resulting IDW refractivity interpolation for airborne SAR applications, all the propagation path losses across Pohang and Heuksando were obtained using the standard atmospheric condition of $\nabla \mathrm{M}=118$ and the observation-based interpolated atmospheric conditions on 15 February 2019. Finally, based on the air-to-ground scenario in the SAR application, two-dimensional illuminated field intensities on the terrain surface were illustrated.

\section{Wheeler Method for Evaluation of Antennas Submerged in Lossy Media}

Oh et al. [12] presented a Wheeler method for the evaluation of the radiation efficiency of submerged antennas within lossy media for the first time in the literature. Extensive investigations have been devised by empirical and simulation methods. A normal-mode helical antenna (NMHA) was first designed and fabricated to exemplify a real-life application at the UHF band ( 0.3 to $3 \mathrm{GHz}$ ). The antenna under test (AUT) was evaluated within an artificial lossy material using a series of Wheeler caps featuring different radii to study the validity of this method. The error between the experimental and simulation radiation efficiency was below $3 \%$ near the theoretical radian length. The presented measurement method of radiation efficiency without any essential measurement facilities or accessories 
could be a promising candidate for fast and accurate evaluation for any wire-type antenna submerged within lossy media.

\section{A Low Profile Dual-Band High Gain Directional Antenna for Anti-Interference WLAN Station Applications}

Dou et al. [13] presented a low-profile dual-band antenna with directional radiation characteristics for wireless local area network (WLAN) applications. The proposed directional antenna was composed of a coupling microstrip line, two F-shaped strips, two rectangular strips, and a defected ground plane. The measured impedance bandwidth of the proposed antenna was $180 \mathrm{MHz}(2.33-2.51 \mathrm{GHz})$ and $830 \mathrm{MHz}(5.09-5.92 \mathrm{GHz})$, which can cover Institute of Electrical and Electronic Engineers (IEEE) $802.11 \mathrm{a} / \mathrm{b} / \mathrm{g}$ frequency bands. The dual-band antenna exhibited desirable directional radiation patterns in the vertical and horizontal planes, with a peak gain of $6.55 \mathrm{dBi}$ in the lower frequency band and $8.1 \mathrm{dBi}$ in the higher frequency band. The measured antenna efficiency was $70 \%$ at $2.4 \mathrm{GHz}$ and $84.5 \%$ at $5.5 \mathrm{GHz}$. The proposed dual-band WLAN station antenna was designed on an FR-4 substrate with overall dimensions of $69 \mathrm{~mm} \times 50 \mathrm{~mm} \times 1.6 \mathrm{~mm}$.

\section{Analysis of the Target Detection Performance of Air-to-Air Airborne Radar Using Long-Range Propagation Simulation in Abnormal Atmospheric Conditions}

Lim et al. [14] proposed the analysis of the target detection performance of air-to-air airborne radars using long-range propagation simulations with a novel quad-linear refractivity model under abnormal atmospheric conditions. The radar propagation characteristics and the target detection performance were simulated using the Advanced Refractive Effects Prediction System (AREPS) software, where the refractivity along the altitude, array antenna pattern, and digital terrain elevation data were considered as inputs to obtain the path loss of the wave propagation. The quad-linear model was used to approximate the actual refractivity data, which were compared to the data derived using the conventional trilinear refractivity model. On the basis of the propagation simulations, a detection performance metric in terms of the atmosphere (DPMA) was proposed for intuitively examining the long-range propagation characteristics of airborne radars in air-to-air situations. To confirm the feasibility of using the DPMA map in various duct scenarios, two actual refractive indices were examined to observe the DPMA results in relation to the height of the airborne radar.

Funding: This research received no external funding.

Acknowledgments: I appreciate the opportunity to organize this Special Issue. Thanks to all authors and peer reviewers, this Special Issue was able to be completed successfully. I would also like to give special thanks to the editorial staff of Applied Sciences for their best support on the publication of this issue.

Conflicts of Interest: The author declares no conflict of interest.

\section{References}

1. Lim, T.H.; Jang, B.J.; Choo, H. Design of heterogenous two-element array antenna on an electrically thick substrate for high isolation and low pattern correlation using modal difference in radiation patterns. Appl. Sci. 2020, 10, 16. [CrossRef]

2. Mneesy, T.S.; Hamad, R.K.; Zaki, A.I.; Ali, W.A.E. A novel high gain monopole antenna array for $60 \mathrm{GHz}$ millimeter-wave communications. Appl. Sci. 2020, 10, 4546. [CrossRef]

3. Jang, D.; Wang, S.; Kim, C.W.; Park, Y.B.; Choo, H. Method for estimating optimal position of S-band relay station through path loss analysis in an outdoor environment. Appl. Sci. 2020, 10, 6089. [CrossRef]

4. Park, S.; Kim, S.; Kim, D.K.; Choi, H.; Jung, K.Y. Numerical study on the feasibility of a 24 GHz ISM-band doppler radar antenna for near-field sensing of human respiration in electromagnetic aspects. Appl. Sci. 2020, 10, 6159. [CrossRef]

5. Radha, S.M.; Jung, M.; Park, P.; Yoon, I.J. Design of an electrically small, planar quasi-isotropic antenna for enhancement of wireless link reliability under NLOS channels. Appl. Sci. 2020, 10, 6204. [CrossRef]

6. Marinho, D.; Arruela, R.; Varun, T.; Matos, J.N. Software-defined radio beamforming system for 5G/radar applications. Appl. Sci. 2020, 10, 7187. [CrossRef] 
7. Youn, S.; Lim, T.H.; Jang, B.J.; Choo, H. Design of a monopulse system using a single patch radiator with a simple multi-mode substrate integrated waveguide feeding network. Appl. Sci. 2020, 10, 7224. [CrossRef]

8. Kim, S.; Choi, J. Quasi-yagi slotted array antenna with fan-beam characteristics for $28 \mathrm{GHz} 5 \mathrm{G}$ mobile terminals. Appl. Sci. 2020, 10, 7686. [CrossRef]

9. Kwon, O.H.; Hwang, K.C. Low-profile Spidron fractal dipole antenna with a ferrite-loaded artificial magnetic conductor for manpack applications. Appl. Sci. 2020, 10, 8843. [CrossRef]

10. Chung, Y. Inverted F type antenna design for the cattle activity and estrus detection sensor module. Appl. Sci. 2021, 11, 298. [CrossRef]

11. Wang, S.; Lim, T.H.; Oh, K.; Seo, C.; Choo, H. Prediction of wide range two-dimensional refractivity using an IDW interpolation method from high-altitude refractivity data of multiple meteorological observatories. Appl. Sci. 2021, 11, 1431. [CrossRef]

12. Oh, Y.; Choi, D.; Lee, J.Y.; Hong, W. Wheeler method for evaluation of antennas submerged in lossy media. Appl. Sci. 2021, 11, 1862. [CrossRef]

13. Dou, Y.; Dong, G.; Lin, J.; Cai, Q.; Liu, G. A low profile dual-band high gain directional antenna for anti-interference WLAN station applications. Appl. Sci. 2021, 11, 2007. [CrossRef]

14. Lim, T.H.; Go, M.; Seo, C.; Choo, H. Analysis of the target detection performance of air-to-air airborne radar using long-range propagation simulation in abnormal atmospheric conditions. Appl. Sci. 2020, 10, 6440. [CrossRef] 